\title{
Association of anesthesia type with prolonged postoperative intubation in neonates undergoing inguinal hernia repair
}

\author{
Abdulraouf Lamoshi $^{1} \cdot$ Jerrold Lerman $^{1,2} \cdot$ Jad Dughayli $^{1,2} \cdot$ Valerie Elberson $^{3} \cdot$ Lorin Towle-Miller $^{4}$. \\ Gregory E Wilding ${ }^{4}$. David H Rothstein ${ }^{1,5}$
}

Received: 15 January 2020 / Revised: 6 May 2020 / Accepted: 22 May 2020 / Published online: 4 June 2020

(c) The Author(s), under exclusive licence to Springer Nature America, Inc. 2020

\begin{abstract}
Purpose The purpose of this study is to determine factors associated with prolonged intubation after inguinal herniorrhaphy in neonates.

Methods Retrospective, single institution review of neonates undergoing inguinal herniorrhaphy between 2010 and 2018. Variables recorded included demographics, comorbidities, ventilation status at time of hernia repair, and anesthetic technique.

Results We identified 97 neonates (median corrected gestational age 39.9 weeks, IQR 6.6). The majority (87.6\%) received general anesthesia (GA); the remainder received caudal anesthesia (CA). Among the GA subjects, $25.8 \%$ remained intubated for at least $6 \mathrm{~h}$ after surgery, whereas none of the CA patients required intubation postoperatively $(p=0.03)$. Two risk factors associated with prolonged postoperative intubation: a history of intubation before surgery $(p=0.04)$ and a diagnosis of bronchopulmonary dysplasia $(p=0.03)$.

Conclusions Neonates undergoing inguinal herniorrhaphy under GA have a greater rate of prolonged postoperative intubation compared with those undergoing CA. A history of previous intubation and bronchopulmonary dysplasia were significant risk factors for prolonged postoperative intubation.
\end{abstract}

\section{Introduction}

Inguinal hernia is the most common medical condition requiring surgery in preterm infants who survived the neonatal period [1]. Twenty to thirty percent of expremature infants who undergo hernia surgery under general anesthesia (GA) experience postoperative apneic episodes [1]. Concerns also exist about the short- and long-

Abdulraouf Lamoshi

alamoshi@kaleidahealth.org

John R. Oishei Children's Hospital, Buffalo, NY, USA

2 Great Lakes Anesthesiology, Buffalo, NY, USA

3 Division of Neonatology and Department of Pediatrics, University at Buffalo Jacobs School of Medicine and Biomedical Sciences, Buffalo, NY, USA

4 Department of Biostatistics, University at Buffalo School of Public Health, Buffalo, NY, USA

5 Department of Surgery, University at Buffalo Jacobs School of Medicine and Biomedical Sciences, Buffalo, NY, USA term neurological sequelae of prolonged anesthetic exposure in young children [2]. Regional anesthesia (RA) is an alternative to GA that is associated with decreased risks of postoperative apnea, periodic breathing, and desaturation, all of which may occur after GA in ex-premature infants [3]. Previously, it was suggested that certain patient factors may increase the risk of developing apnea, neurodevelopmental change, and respiratory and circulatory complications after GA $[4,5]$. These factors included gestational age at birth, post-conceptional age at the time of surgery, size for gestational age, and anemia [4]. However, previous studies have not explored an association between the preoperative comorbidities and the duration of postoperative intubation.

Neuromuscular blocker drugs (NMBDs) play an important role in facilitating tracheal intubation and mechanical ventilation in neonates and infants. However, if the neuromuscular blockade is not antagonized, the effects of NMBDs may increase the duration of postoperative intubation. Effects of NMBDs including pulmonary complications may extend beyond the completion of surgery in as many as $88 \%$ of infants [6]. Alternately, agents that antagonize the effects of NMBDs, such neostigmine, can 
restore normal muscle tone and strength, and abbreviate the duration of postoperative intubation [6].

The aim of this study was to determine whether the type of anesthesia or neonatal preoperative medical conditions is associated with prolonged postoperative tracheal intubation after inguinal herniorrhaphy in neonates. We also sought to determine the impact of NMBDs and agents that antagonize their effects on the duration of postoperative tracheal intubation.

\section{Methods}

This was a retrospective single institution chart review of neonates after inguinal herniorrhaphy between 2010 and 2018. The University at Buffalo Institutional Review Board approved this study, study approval number: 2849 . Those whose airways were intubated preoperatively were excluded from the analysis.

Several variables were recorded, including demographics, respiratory comorbidities such as respiratory distress syndrome, the presence of persistent pulmonary hypertension, bronchopulmonary dysplasia (BPD), and any other respiratory problems, major and minor cardiac defects, renal and liver problems, atrial/brain natriuretic peptide levels, steroid use, history of prior intubation, use of NMBDs and opioids, neonatal apnea, ongoing apnea, anemia, GA vs. caudal anesthesia (CA) modality, and conversion from caudal to general, and the use of agents postoperatively to antagonize neuromuscular blockade. Major cardiac defects were defined as any congenital heart anomaly that requires surgical interventional or cardiac catheterization before discharge from the neonatal intensive care unit (NICU). The patients that converted from CA to GA were included in the GA group. In addition, postoperative data including the time from the conclusion of anesthesia until tracheal extubation were categorized into two groups: immediate $(<6 \mathrm{~h})$ and late extubation $(\geq 6 \mathrm{~h})$. Lastly, we recorded all reintubations during the subsequent $72 \mathrm{~h}$.

All postoperative complications, including cardiac arrest, aspiration, inhaled nitric oxide use postoperatively, pulmonary edema, anaphylactic shock, drug overdose, severe arrhythmia, respiratory depression, complication of intubation, and death, as well as surgical complications were recorded. Fixed inspiratory oxygen levels before and after operation, and postoperative ventilation mode and peak pressure were documented. In this data collection, the primary outcome was prolonged postoperative intubation, defined as $\geq 6 \mathrm{~h}$ after surgery.

Statistical analysis was completed using SAS version 9.4. Fisher's exact test, Wilcoxon's signed-rank test, and logistic regression were utilized to compare GA and CA, as well as immediate vs. late intubation. For continuous data, we used Wilcoxon's rank-sum test, because the data were not normaly distributed. Also, the association between the preoperative comorbidities and the postoperative intubation time was explored. Finally, an association between the use of agents to antagonize NMBDs and the duration of tracheal intubation was examined using Fisher's exact test and the corresponding odds ratios were reported. A nominal significance level of 0.05 was used on all statistical tests.

\section{Results}

We reviewed the charts of 97 neonates ( $85 \%$ male, median corrected gestational age 39.9 weeks, interquartile range 6.6). A total of 85 neonates $(87.6 \%)$ received GA; the remainder received CA. The type of anesthesia was chosen by the pediatric anesthesiologist in concert with the attending pediatric surgeon. The airways in all neonates who received GA were intubated for surgery. In 18 cases $(18.5 \%)$, CA was the practitioner's first choice, although one-third (6/18) of the CA cases were converted to GA to complete the surgery. CA comprised $1 \%$ lidocaine with epinephrine or 3\% 2-chloroprocaine. The main reasons for the conversion from CA to GA were hypoxemia, bradycardia, disruptive movement during surgery, or dural puncture during placement of the CA.

Demographic data, comorbidities, length of stay, and pre-op or postop $\mathrm{FiO}_{2}$ concentrations in the two groups were similar (Table 1). Among those who received GA, $32.9 \%(28 / 85)$ remained intubated for at least $6 \mathrm{~h}$ after surgery, whereas $0 \%$ of those who received CA required intubation at any time $(p=0.03)$. Missing data were limited to some postoperative variables in 15 patients $(15.5 \%)$.

Fisher's exact test yielded two risk factors that were significantly associated with prolonged postoperative intubation: a history of tracheal intubation before surgery $(p=$ $0.04)$ and a diagnosis of BPD $(p=0.03)$. The frequency of postoperative hernia-specific complications was similar in the two groups.

Among the 25 neonates who remained intubated $\geq 6 \mathrm{~h}$ postoperatively, $3(12 \%)$ were extubated between 6 and $12 \mathrm{~h}, 11(44 \%)$ between 12 and $24 \mathrm{~h}$, and 11 (44\%) after more than $24 \mathrm{~h}$. After excluding missing data, $81.9 \%$ (59/ 72) of the GA group received NMBDs, $22 \%$ (13/59) of whom received agents to antagonize the blockade postoperatively. Only 2 of these $13(15.4 \%)$ remained intubated for $\geq 6 \mathrm{~h}$. The use of agents to antagonize NMBDs was inversely related to prolonged intubation (odds ratio $=0.20$, $p=0.05$ ). Prolonged postoperative intubation time was significantly associated with the preoperative and postoperative $\mathrm{FiO}_{2}(p<0.001)$ (Table 2). 
Table 1 Demographics and preoperative comorbidities of GA and CA groups.

\begin{tabular}{llll}
\hline Variable & GA & CA & $p$ \\
\hline Adjusted age at time of surgery & 42.3 Weeks & 39.1 Weeks & 0.76 \\
Weight at time of surgery & $3.2 \mathrm{~kg}$ & $2.5 \mathrm{~kg}$ & 0.07 \\
PHT & $10(11.8 \%)$ & $1(8.3 \%)$ & 1.00 \\
BPD & $55(64.7 \%)$ & $7(58.3 \%)$ & 0.75 \\
RDS & $61(73.5 \%)$ & $7(58.3 \%)$ & 0.31 \\
ORD & $58(68.2 \%)$ & $7(58.3 \%)$ & 0.52 \\
Major CHD & $7(8.2 \%)$ & $0(0 \%)$ & 0.59 \\
Minor CHD & $67(78.8 \%)$ & $7(58.3 \%)$ & 0.15 \\
Prior intubation & $67(79.8 \%)$ & $9(75 \%)$ & 0.71 \\
Muscle relaxants use & $13(17.8 \%)$ & $0(0 \%)$ & 0.35 \\
Opioid use & $74(87.1 \%)$ & $10(83.3 \%)$ & 0.66 \\
Neonatal apnea & $59(69.4 \%)$ & $6(50 \%)$ & 0.20 \\
Ongoing apnea & $58(68.2 \%)$ & $6(50 \%)$ & 0.33 \\
Anemia & $78(91.8 \%)$ & $12(100 \%)$ & 0.59 \\
Steroids use & $62(72.9 \%)$ & $8(66.7 \%)$ & 0.73 \\
Renal problems & $43(50.6 \%)$ & $5(41.7 \%)$ & 0.76 \\
Liver problems & $65(76.5 \%)$ & $11(91.7 \%)$ & 0.45 \\
\hline BPD bronchopu &
\end{tabular}

$B P D$ bronchopulmonary dysplasia, $C A$ caudal anesthesia, $C H D$ congenital heart disease, $G A$ general anesthesia, $O R D$ other respiratory disorders, $P H T$ pulmonary hypertension, $R D S$ respiratory distress syndrome.

Table 2 Demographics and preoperative comorbidities of prolonged and short-no intubation groups.

\begin{tabular}{|c|c|c|c|}
\hline Variable & PIG & SNIG & $p$ \\
\hline Adjusted age at time of surgery & 41.8 Weeks & 42.0 Weeks & 0.53 \\
\hline Weight at time of surgery & $3.2 \mathrm{~kg}$ & $3.1 \mathrm{~kg}$ & 0.08 \\
\hline PHT & $6(21.4 \%)$ & $4(7.0 \%)$ & 0.07 \\
\hline BPD & $23(82.1 \%)$ & $32(56.1 \%)$ & $\mathbf{0 . 0 3}$ \\
\hline RDS & $22(84.6 \%)$ & $39(68.4 \%)$ & 0.18 \\
\hline ORP & $23(82.1 \%)$ & $35(61.4 \%)$ & 0.08 \\
\hline Major CHD & $4(14.3 \%)$ & $3(5.3 \%)$ & 0.21 \\
\hline Minor CHD & $23(82.1 \%)$ & $44(77.2 \%)$ & 0.78 \\
\hline Prior intubation & $26(92.9 \%)$ & $41(73.2 \%)$ & 0.04 \\
\hline Muscle relaxants use & $2(7.7 \%)$ & $11(23.4 \%)$ & 0.12 \\
\hline Opioid use & $27(96.4 \%)$ & $47(82.5 \%)$ & 0.09 \\
\hline Neonatal apnea & $20(71.4 \%)$ & $39(68.4 \%)$ & 1.00 \\
\hline Ongoing apnea & $19(67.9 \%)$ & $39(68.4 \%)$ & 1.00 \\
\hline Anemia & $28(100 \%)$ & $50(87.7 \%)$ & 0.09 \\
\hline Steroids use & $22(78.6 \%)$ & $40(70.2 \%)$ & 0.45 \\
\hline Renal problems & $18(64.3 \%)$ & $25(43.9 \%)$ & 0.11 \\
\hline Liver problems & $24(85.7 \%)$ & $41(71.9 \%)$ & 0.19 \\
\hline Preoperative $\mathrm{FiO} 2$ & 51.0 & 29.2 & $<0.001$ \\
\hline Postoperative $\mathrm{FiO} 2$ & 36.9 & 30.7 & $<0.001$ \\
\hline
\end{tabular}

$B P D$ bronchopulmonary dysplasia, $C H D$ congenital heart disease, $O R D$ other respiratory disorders, $P H T$ pulmonary hypertension, $P I G$ prolonged intubation group, RDS respiratory distress syndrome, $S N I G$ short-no intubation group, $P$-values in bold indicate statistically significant results.
Intent-to-treat analysis demonstrated no significant difference in preoperative comorbidities between the 18 neonates selected for CA and the 79 neonates selected for GA. Compared with the immediate extubation group $(n=59)$, those whose airways were intubated for more than one day (11) postoperatively required significantly greater levels of $\mathrm{FiO}_{2}$ both pre- and postoperatively, an average of 26 vs. 42 $(p=0.004)$.

\section{Intubation time analysis (raw times)}

Given that the sample size is a little small for this group, we applied basic tests. For the continuous covariates, we generated a spearman correlation and corresponding $p$-values to determine whether there was a relationship between the covariate and intubation time. We found that only birth weight and weight at the time of surgery were significantly associated with prolonged intubation $(P=0.04$ and $p=$ 0.02 ) respectively, i.e., as the weight of the neonates increased the duration of intubation decreased $(r=-0.42$ and $r=-0.5)$, respectively. We also generated the mean intubation time across the categorical covariates and corresponding Wilcoxon signed-rank test for differences, but none of those were significant.

\section{Power analysis}

Typically, a power analysis would be done prior to data collection to determine a general sample size, but our study was limited from the start to the data that already have access to, a power analysis would not necessarily be suited here. With a sample size of 85 and 12, estimated proportions corresponding to dichotomous variables could have a maximum standard error of 0.114 and 0.054 , respectively. Of the GA subjects, 25 subjects had long intubation and 60 did not have long intubation. The estimated proportions corresponding to dichotomous variables within the 25 prolonged intubated neonates vs. the 60 immediate intubated groups could have a maximum SE of 0.096 and 0.066 , respectively.

\section{Discussion}

In a large cohort of neonates undergoing inguinal herniorrhaphy surgery, we found a predictably larger fraction of postoperative tracheal intubation in the GA group when compared with those undergoing CA. A history of previous intubation and BPD significantly predicted prolonged postoperative intubation, whereas agents that antagonize NMBDs abbreviated the duration of postoperative intubation. These results are significant in highlighting the importance of the mode of anesthesia and preoperative 
comorbidities on the duration of postoperative tracheal intubation in neonates undergoing inguinal hernia surgery.

There is a paucity of published data regarding outcomes after hernia repair in neonatal patients in terms of their need for mechanical ventilation support based on their preoperative status. Previous studies reported a number of factors that contribute to postoperative apnea. For example, premature infants less than 44 weeks post-conceptional age and those who are anemic are at increased risk for post-anesthetic apnea compared with infants older than 44 weeks post-conceptional age $[7,8]$. Post-conceptional size for gestational age, BPD, necrotizing enterocolitis, and a history of apnea or ongoing apnea are additional factors that are reported to increase the risk of post-anesthetic apnea $[4,9,10]$. Nonetheless, none of these studies explored the frequency of complications in neonates who required prolonged intubation. RA (spinal/ caudal) is a technique that could be offered to critically ill neonates who may face daunting challenges if they received GA and tracheal intubation. However, this study and similar previous studies found that GA and RA were being used in similar cohorts regardless of demographics and preoperative comorbidities [11]

One of the key findings from this study is that BPD, which is defined as a consistent requirement of supplemental oxygen $(>21 \%)$ at 36 weeks corrected gestation, is significantly associated with prolonged postoperative mechanical ventilation. The association for BPD, postanesthetic apnea, and need for postoperative mechanical ventilation was observed previously suggesting that BPD could lead to post-anesthetic apnea because of the presence of interstitial fibrosis and fluid retention [10, 11].

Although not definitively described in the literature, prolonged postoperative intubation has been strongly associated with a history of prior intubation. This observation could be related to the severity of the underlying disease that required mechanical ventilation in the first place or to upper airway anatomic trauma caused by the previous intubation. In adults, vocal cord granulomas were reported bilaterally in about half of the patients with prior intubations, taking up to 70 weeks before the patients went into remission [12]. Neonatal anesthesia has traditionally utilized uncuffed tracheal tubes to minimize airway mucosal injury [13]. However, a review study concerning injury after tracheal intubation in neonates concluded that tracheal intubation can lead to both glottic and subglottic inflammation and stenosis, and that the severity of the injury increases in proportion to the duration of tracheal intubation [14]. Hence, the duration of tracheal intubation in neonates should be limited. It is worth mentioning that prolonged intubation can increase risk of reintubation, subglottic stenosis, laryngeal injury, ventilator-associated pneumonia/ events, swallowing and speech impairment, and tracheobronchitis [14].
Concern has been expressed previously that the use of NMBDs in neonates can lead to residual neuromuscular effects that may include postanesthesia hypoxia, airway obstruction, the need for reintubation, impaired respiratory response to hypoxia, and decreased forced vital capacity $[6,15]$. Residual neuromuscular blockade may result from any of several possible reasons including the dose of NMBDs during surgery in relation to the duration of the surgery and the lack of neuromuscular blockade monitoring and the failure to antagonize the neuromuscular blockade at the conclusion of surgery [16]. Although the use of agents to antagonize the neuromuscular blockade varied inversely with the duration of prolonged intubation and are recommended to ensure the integrity of the neuromuscular junction before tracheal extubation [15, 17], only $15 \%$ of the neonates in whom neuromuscular blockade was used received an anticholinesterase, neostigmine. Sugammadex is a distinctive neuromuscular reversal agent that belongs to a new selective relaxant class; it reverses the aminosteroid non-depolarizing muscle relaxants (rocuronium and vecuronium) [18]. With the introduction of Sugammadex into clinical practice, complete antagonism of steroidal neuromuscular blocking drugs such as rocuronium may be achieved even after large doses of rocuronium during relative brief surgery in neonates and children [18, 19]. Although not approved by the Food and Drug Administration for use in neonates, the available experience suggests that Sugammadex will completely antagonize neuromuscular blockade in neonates, despite the immature development of the neuromuscular junction in this age group [20]. A large case-controlled study concluded that antagonizing the NMBD effects could effectively reduce the morbidity and mortality after anesthesia and we posit this may be extended to neonates [21].

Although the conversion rate from CA to GA in this study was large, it is consistent with rates $(3.9 \%-23.9 \%)$ reported in prior studies $[22,23]$. We routinely use CA instead of spinal anesthesia for lower abdominal surgery in this population to provide supplemental local anesthesia in the caudal block if the surgery is prolonged and to avoid the failure rate associated with spinal anesthesia [22]. The main contributing factors for the conversion from CA to GA include technical difficulties from performing the caudal block, unexpected surgical difficulties, a vigorously crying patient, surgeon preference, and repeated apnea [10, 24]. Interestingly, there was no significant difference between the immediate and prolonged intubation groups with the intent to treat analysis. However, as there is no clear guidelines/indications for which neonates would receive CA vs. GA, we believe that these six patients were most appropriately analyzed in the GA group.

This study has several limitations, which may limit its generalizability. First, missing data is a major hurdle with any retrospective study. Second, the sample size particularly 
for the CA group was small, considering the commonality of the inguinal hernia. Third, a history of prior intubation could be attributed to multiple factors, rendering the practical interpretation of this finding a serious challenge. Alternately, the association of prolonged intubation with a prior history of intubation demonstrates that any underlying cause that was managed by mechanical ventilation is a potential cause for postoperative prolonged intubation. In spite of these limitations, the chief strength of this study is that it considered a wide range of preoperative comorbidities, to predict the risk of postoperative prolonged intubation for neonates with inguinal hernias who undergo a herniotomy during their NICU stay. Of note, we did not categorize the cases based on the surgical approach (laparoscopic versus open), because we believe that laparoscopic hernia repair is not necessarily faster, less painful, or cosmetically superior to the open approach; at least not to that degree as described in some studies. We also have a bias to request CA for the "sicker" neonates because we believe RA impacts the cardiopulmonary system to a lesser extent than GA. However, our statistical analysis failed to identify any significant difference between the CA and GA groups in terms of preoperative comorbidities, including BPD and a history of intubation.

\section{Conclusion}

CA is associated with a substantially decreased need for postoperative tracheal intubation when compared with GA in neonates undergoing inguinal herniorrhaphy. History of prior intubation and/or BPD may jeopardize the postoperative course for neonatal patients after herniorrhaphy by increasing the need for postoperative ventilation support. In addition, using agents that antagonize neuromuscular blockade may reduce the duration of postoperative tracheal intubation.

Acknowledgements Statistical analysis reported in this publication was supported by the National Center for Advancing Translational Sciences of the National Institutes of Health under award Number UL1TR001412. The content is solely the responsibility of the authors and does not necessarily represent the official views of the NIH.

\section{Compliance with ethical standards}

Conflict of interest The authors declare that they have no conflict of interest.

Publisher's note Springer Nature remains neutral with regard to jurisdictional claims in published maps and institutional affiliations.

\section{References}

1. Craven PD, Badawi N, Henderson-Smart DJ, Obrien M. Regional (spinal, epidural, caudal) versus general anaesthesia in preterm infants undergoing inguinal herniorrhaphy in early infancy. Cochrane Database Syst. Rev. 2003. https://doi.org/10.1002/ 14651858.cd003669

2. Mccann ME, Soriano SG. General anesthetics in pediatric anesthesia: influences on the developing brain. Curr Drug Targets. 2012;13:944-51. https://doi.org/10.2174/138945012800675768

3. Krane EJ, Haberkern CM, Jacobson LE. Postoperative apnea, bradycardia, and oxygen desaturation in formerly premature infants. Anesthesia Analgesia. 1995;80:7-13. https://doi.org/10. 1097/00000539-199501000-00003

4. Cote CJ, Zaslavsky A, Downes JJ, Kurth CD, Welborn LG, Warner LO, et al. Postoperative apnea in former preterm infants after inguinal herniorrhaphy. Anesthesiology. 1995;82:809-22. https://doi.org/10.1097/00000542-199504000-00002

5. Tiret L, Nivoche Y, Hatton F, Desmonts J, Vourc HG. Complications related to anaesthesia in infants and children. $\mathrm{Br} \mathrm{J}$ Anaesth. 1988;61:263-9. https://doi.org/10.1093/bja/61.3.263

6. Zafirova Z, Dalton A. Neuromuscular blockers and reversal agents and their impact on anesthesia practice. Best Pract Res Clin Anaesthesiol. 2018;32:203-11. https://doi.org/10.1016/j.bpa. 2018.06.004

7. Malviya S, Swartx J, Lerman J. Are all preterm infants younger than 60 weeks postconceptual age at risk for postanesthetic apnea? Anesthesiology. 1993;78:1076-81. https://doi.org/10.1097/ 00000542-199306000-00009

8. Welborn LG, Hannallah RS, Higgins T, Fink R, Luban N. A1091 Does anemia increase the risk of postoperative apnea in former preterm infants? Anesthesiology 1990;73. https://doi.org/10.1097/ 00000542-199009001-01089

9. Lynn A. Faculty of 1000 evaluation for apnea after awake regional and general anesthesia in infants: the general anesthesia compared to spinal anesthesia study-comparing apnea and neurodevelopmental outcomes, a randomized controlled trial. F1000 - PostPublication Peer Review of the Biomedical Literature 2015. https://doi.org/10.3410/f.725508900.793507986

10. Gerber AC, Weiss M. Awake spinal or caudal anaesthesia in preterms for herniotomies: what is the evidence based benefit compared with general anaesthesia? Curr Opin Anaesthesiol. 2003;16:315-20. https://doi.org/10.1097/00001503-200306000-00012

11. Somri M, Gaitini L, Vaida S, Collins G, Sabo E, Mogilner G. Postoperative outcome in high-risk infants undergoing herniorrhaphy: comparison between spinal and general anaesthesia. Anaesthesia. 1998;53:762-6. https://doi.org/10.1046/j.1365-2044. 1998.00431.x

12. Franco R. Vocal process granuloma - a result of long-term observation in 53 patients. Yearb Otolaryngol-Head Neck Surg. 2010;2010:70-1. https://doi.org/10.1016/s1041-892x(10)79564-0

13. Bhardwaj N. Pediatric cuffed endotracheal tubes. J Anaesthesiol Clin Pharmacol. 2013;29:13 https://doi.org/10.4103/0970-9185. 105786

14. Wei JL, Bond J. Management and prevention of endotracheal intubation injury in neonates. Curr Opin Otolaryngol Head Neck Surg. 2011;19:474-7. https://doi.org/10.1097/moo.0b013e32834c7b5c

15. Souza CMD, Romero FE, Tardelli MA. Assessment of neuromuscular blockade in children at the time of block reversal and the removal of the endotracheal tube. Braz J Anesthesiol. 2011;61:145-55. https://doi.org/10.1016/s0034-7094(11)70019-8

16. Fortier L-P, Mckeen D, Turner K, Médicis ÉD, Warriner B, Jones $\mathrm{PM}$, et al. The RECITE study. Anesthesia Analgesia. 2015;121:366-72. https://doi.org/10.1213/ane.0000000000000757

17. Meretoja OA. Neuromuscular block and current treatment strategies for its reversal in children. Pediatr Anesthesia. 2010;20:591-604. https://doi.org/10.1111/j.1460-9592.2010.03335.x

18. Carlos RV, Torres MLA, Boer HDD. Rocuronium and sugammadex in a 3 days old neonate for draining an ovarian cyst. Neuromuscular management and review of the literature. Braz 
J Anesthesiol (Engl Ed). 2016;66:430-2. https://doi.org/10.1016/j. bjane.2015.01.004

19. Liu G, Wang R, Yan Y, Fan L, Xue J, Wang T. The efficacy and safety of sugammadex for reversing postoperative residual neuromuscular blockade in pediatric patients: a systematic review. Scientific Reports 2017;7. https://doi.org/10.1038/s41598-017-06159-2

20. Anderson BJ, Larsson P, Lerman J. Anesthesia and ancillary drugs and the neonate. Neonatal Anesthesia 2014:67-130. https://doi. org/10.1007/978-1-4419-6041-2_3

21. Mcgoldrick KE. Impact of anesthesia management characteristics on severe morbidity and mortality. Surv Anesthesiol. 2005;49:140 https://doi.org/10.1097/01.sa.0000165233.61632.ab
22. Frawley G, Bell G, Disma N, Withington DE, Graaff JCD, Morton NS, et al. Predictors of failure of awake regional anesthesia for neonatal hernia repair. Anesthesiology. 2015; 123:55-65. https://doi.org/10.1097/aln.0000000000000708

23. Hoelzle M, Weiss M, Dillier C, Gerber A. Comparison of awake spinal with awake caudal anesthesia in preterm and ex-preterm infants for herniotomy1. Pediatr Anesthesia. 2010;20:620-4. https://doi.org/10.1111/j.1460-9592.2010.03316.x

24. Maitra S, Baidya DK, Pawar DK, Arora MK, Khanna P. Epidural anesthesia and analgesia in the neonate: a review of current evidences. J Anesthesia. 2014;28:768-79. https://doi.org/10.1007/ s00540-014-1796-8 